\title{
Identification and verification of differentially expressed microRNAs and their target genes for the diagnosis of esophageal cancer
}

\author{
XIAOYUE CAI $^{1 *}$, XIWEN YANG ${ }^{1 *}$, CHANGJUAN JIN $^{1}$, LEI LI $^{1}$, QING CUI $^{1}$, YIJUN GUO ${ }^{1}$, \\ YUN DONG $^{1}$, XIAOHUA YANG ${ }^{2}$, LILI GUO $^{2}$ and MING ZHANG ${ }^{1}$ \\ ${ }^{1}$ Department of Integrated Traditional Chinese Medicine and Western Medicine; \\ ${ }^{2}$ Research Center, Shanghai Chest Hospital, Shanghai Jiaotong University, Shanghai 200030, P.R. China
}

Received October 12, 2016; Accepted January 11, 2018

DOI: $10.3892 / \mathrm{ol} .2018 .9066$

\begin{abstract}
Bioinformatic tools were used to analyze GSE6188, GSE13937 and GSE43732 microarrays, and the top 10 upregulated and downregulated genes of each microarray were identified. It was determined that human microRNA (hsa-miR)-1 and hsa-miR-203 were two downregulated genes in common. Subsequently, it was identified that there were 145 and 335 genes in common targeted by hsa-miR-1 and hsa-miR-203, respectively. In order to narrow the number of target genes down further, the target genes were compared with GSE26886 microarray data. There were five upregulated genes in common with hsa-miR-1, i.e., MMD, BICD1, PTPRG, SDC2 and SEMA6D, and there were eight upregulated genes in common with hsa-miR-203, i.e., PXDN, NRCAM, FMNL2, EIF5A2, GLI3, FSL1, GREM1 and AHR. These genes may become promising biomarkers for the diagnosis of esophageal cancer.
\end{abstract}

\section{Introduction}

There are two main subtypes of esophageal cancer: Esophageal squamous cell carcinoma (ESCC, ac counting for between 60 and $70 \%$ of cases), which is more common in the developing world, and esophageal adenocarcinoma (EAC, accounting for between 20 and $30 \%$ of cases), which is more common in the developed world (1). Symptoms of esophageal cancer include difficulty in swallowing (accompanied by pain), weight loss, a hoarse voice, enlarged lymph nodes (glands) around the collar

Correspondence to: Dr Ming Zhang, Department of Integrated Traditional Chinese Medicine and Western Medicine, Shanghai Chest Hospital, Shanghai Jiaotong University, 241 Huaihai West Road, Shanghai 200030, P.R. China

E-mail: gason2000@126.com

*Contributed equally

Key words: esophageal cancer, biomarker, hsa-miR-1, hsa-miR-203 bone, a dry cough, and possibly coughing up or vomiting blood (2).

As of 2012, esophageal cancer is the eighth most common cancer globally with 456,000 novel cases annually (3). It has become the sixth most common cause of cancer-associated mortality owing to its poor prognosis, with 5-year survival rates of between 13 and $18 \%$ (4). The high mortality rate of patients with esophageal cancer is a result of the difficulty of its detection in its early stages (4). Therefore, it has become a global public health issue and a burden on healthcare systems. In order to address this issue, identification of more comprehensive and accurate biomarker genes is required for early diagnosis.

MicroRNAs (miRNAs/miRs) are small non-coding RNAs containing 22 nucleotides, which serve important functions in RNA silencing and post-transcriptional regulation of gene expression $(5,6)$. It has been identified that the expression level of miRNAs may be used for determining prognosis. For instance, a previous study on non-small cell lung cancer samples revealed that low miR-324a levels may serve as a prognostic indicator of poor survival (7). Either high miR-185 or low miR-133b levels may be associated with metastasis and poor survival in colorectal cancer (8). In classical Hodgkin's lymphoma, plasma miR-21, miR-494 and miR-1973 are promising disease response biomarkers (9). miR-205 was identified previously as a target for inhibiting metastasis of breast cancer (10). A total of five members of the miR-200 family (miR-200a, miR-200b, miR-200c, miR-141 and miR-429) are downregulated in tumor progression of breast cancer (11).

Accordingly, the aim of the present study was to identify miRNA markers that may serve as early diagnostic markers for esophageal cancer. In the present study, differentially expressed genes in esophageal cancer tissue compared with normal esophageal tissue in common in the GSE6188, GSE13937 and GSE43732 microarrays were identified. Subsequently, the expression of these genes was verified, and the target genes of these genes using the TargetScan $(12,13)$, miRDB $(14,15)$ and PITA (16) databases were identified to potentially reveal more detailed and precise diagnostic markers for esophageal cancer. 


\section{Materials and methods}

GSE microarray data analysis. In total, three sets of gene expression profile data of patients with esophageal cancer were downloaded from the Gene Expression Omnibus (GEO) database (www.ncbi.nlm.nih.gov/geo): GSE6188 (257 samples), GSE13937 (152 samples) and GSE43732 (238 samples), all representing adenocarcinoma. The GSE43732 microarray database (Agilent-038166 cbc_human_miR18.0) contains 119 esophageal cancer tissue samples and 119 para-carcinoma tissue samples. The GSE6188 microarray database (Tsinghua University mammalian 2K miRNA microarray) contains 153 esophageal cancer tissue samples and 104 para-carcinoma tissue samples. The GSE13937 microarray database (Ohio State University Comprehensive Cancer Center Human and Mouse MicroRNA Microarray version 3.0) contains 76 esophageal cancer tissue samples and 76 para-carcinoma tissue samples. In addition, GSE26886 consisted of samples from 19 healthy subjects, 20 specimens from patients with Barrett's esophagus, 30 cases of esophageal adenocarcinoma and 9 cases of esophageal squamous cell carcinoma. The comparison analysis was between 19 healthy subjects and subjects with other tumors. On the basis of the three GSE databases, following removal of the redundant and unannotated sequences with a false discovery rate (FDR) $<1 \%$, upregulated and downregulated miRNAs with fold-changes $>2$, respectively, were identified.

Screening differentially expressed genes and identifying their target genes. In order to identify the differentially expressed miRNAs common to the microarrays used, Venny (version 2.0.2; Centro Nacional de Biotechnología; bioinfogp.cnb.csic.es/tools/venny/index.html) was employed. Subsequently, TargetScan, miRDB and PITA online databases were used to predict the target genes of these miRNAs, and identify the target genes in common using Venny.

Protein interaction network. The Human Protein Reference Database (HPRD; www.hprd.org) was used to build a human protein interaction network, and observe the distribution of the target genes of the differentially expressed miRNAs to identify the molecular basis of esophageal carcinoma.

Statistical analysis. All statistical analyses were performed using SPSS software (version 19.0; IBM Corp., Armonk, NY, USA). An independent samples t-test was used to compare the difference between two groups. $\mathrm{P}<0.05$ was considered to indicate a statistically significant difference.

\section{Results}

Differentially expressed genes in the GSE6188 microarray. As presented in Fig. 1, 153 esophageal cancer tissues and 104 para-carcinoma tissues were analyzed, following removal of the redundant and unannotated sequences, with a FDR $<1 \%$. A total of 93 genes were identified to be significantly upregulated (fold-change $>2$ ) and 10 genes were identified to be significantly downregulated (fold-change $>2$ ) in esophageal cancer tissues. The top 10 upregulated and downregulated genes are presented in Table I.
Table I. Top 10 upregulated and downregulated genes in esophageal cancer tissue based on the GSE6188 microarray.

\begin{tabular}{lcc} 
A, Upregulation & & \\
\hline Name & Log (fold-change) & P-value \\
\hline has-miR-196a & 0.902872 & $1.39 \times 10^{-23}$ \\
hsa-miR-7 & 0.850074 & $5.74 \times 10^{-23}$ \\
hsa-miR-196b & 0.737501 & $2.92 \times 10^{-18}$ \\
hsa-miR-424 & 0.732302 & $4.14 \times 10^{-23}$ \\
hsa-miR-503 & 0.663847 & $6.42 \times 10^{-25}$ \\
hsa-miR-301 & 0.65897 & $1.30 \times 10^{-19}$ \\
rno-miR-7 & 0.65262 & $9.39 \times 10^{-13}$ \\
hsa-miR-183 & 0.573863 & $1.79 \times 10^{-19}$ \\
hsa-miR-493 & 0.558956 & $2.24 \times 10^{-13}$ \\
hsa-miR-182 & 0.558531 & $6.70 \times 10^{-11}$ \\
\hline
\end{tabular}

\section{B, Downregulation}

\begin{tabular}{lcc}
\hline Name & Log (fold-change) & P-value \\
\hline hsa-miR-375 & -0.87446 & $2.51 \times 10^{-20}$ \\
hsa-miR-133b & -0.67629 & $5.74 \times 10^{-16}$ \\
hsa-miR-133a & -0.62342 & $2.21 \times 10^{-13}$ \\
hsa-miR-1 & -0.53102 & $1.05 \times 10^{-8}$ \\
hsa-miR-203 & -0.37068 & $4.14 \times 10^{-9}$ \\
hsa-miR-497 & -0.37053 & $1.44 \times 10^{-9}$ \\
hsa-miR-150 & -0.35903 & $3.16 \times 10^{-9}$ \\
rno-miR-140 & -0.32767 & $8.92 \times 10^{-9}$ \\
hsa-miR-30a-3p & -0.32656 & $8.13 \times 10^{-6}$ \\
hsa-miR-126 & -0.30137 & 0.000172 \\
\hline
\end{tabular}

miR, microRNA; hsa, human; rno, rat.

Differentially expressed genes in the GSE13937 microarray. As presented in Fig. 2, carcinoma tissues and para-carcinoma tissues from 43 patients with ESCC and 32 patients with EAC were analyzed. In total, 18 patients were identified as Barrett's esophagus which was associated with early esophageal cancer and carcinoma of gastric cardia. The differentially expressed miRNAs in esophageal cancer tissue compared with para-carcinoma tissues based on the GSE13937 microarray were identified. Following removal of the redundant and unannotated sequences with a FDR $<1 \%, 68$ genes were identified to be significantly upregulated (fold-change $>2$ ) and 27 genes were identified to be significantly downregulated (fold-change $>2$ ) in esophageal cancer tissues. The top 10 upregulated and downregulated genes are presented in Table II.

Differentially expressed genes in the GSE43732 microarray. As presented in Fig. 3, 119 esophageal cancer tissues and para-carcinoma tissues were analyzed, following removal of the redundant and unannotated sequences, with a FDR $<1 \%$. In total, 101 genes were identified to be significantly upregulated (fold-change $>2$ ) and 171 genes were identified to be significantly downregulated (fold-change $>2$ ) in esophageal cancer 


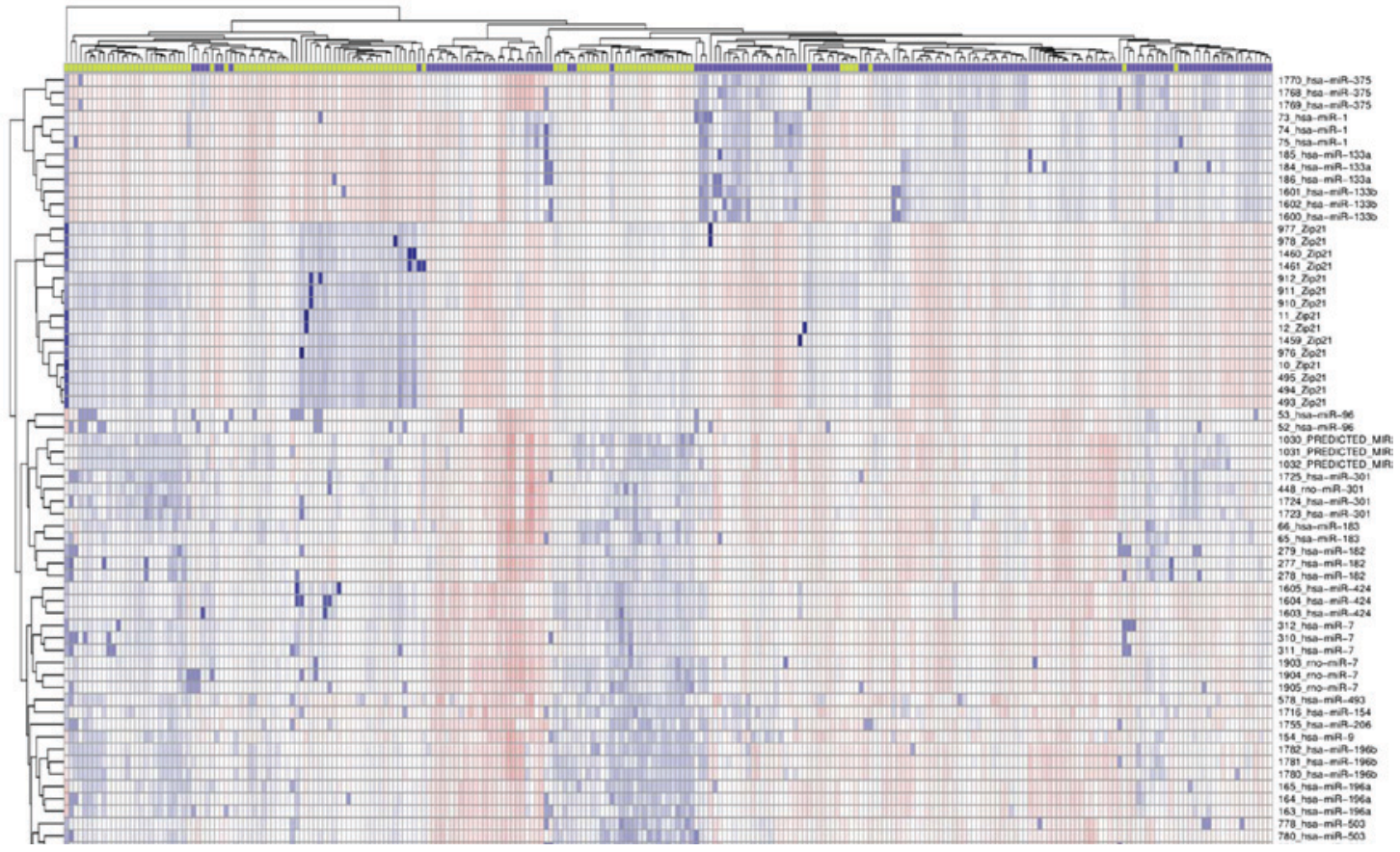

Figure 1. Heat map of the differentially expressed genes of esophageal cancer based on the GSE6188 microarray.

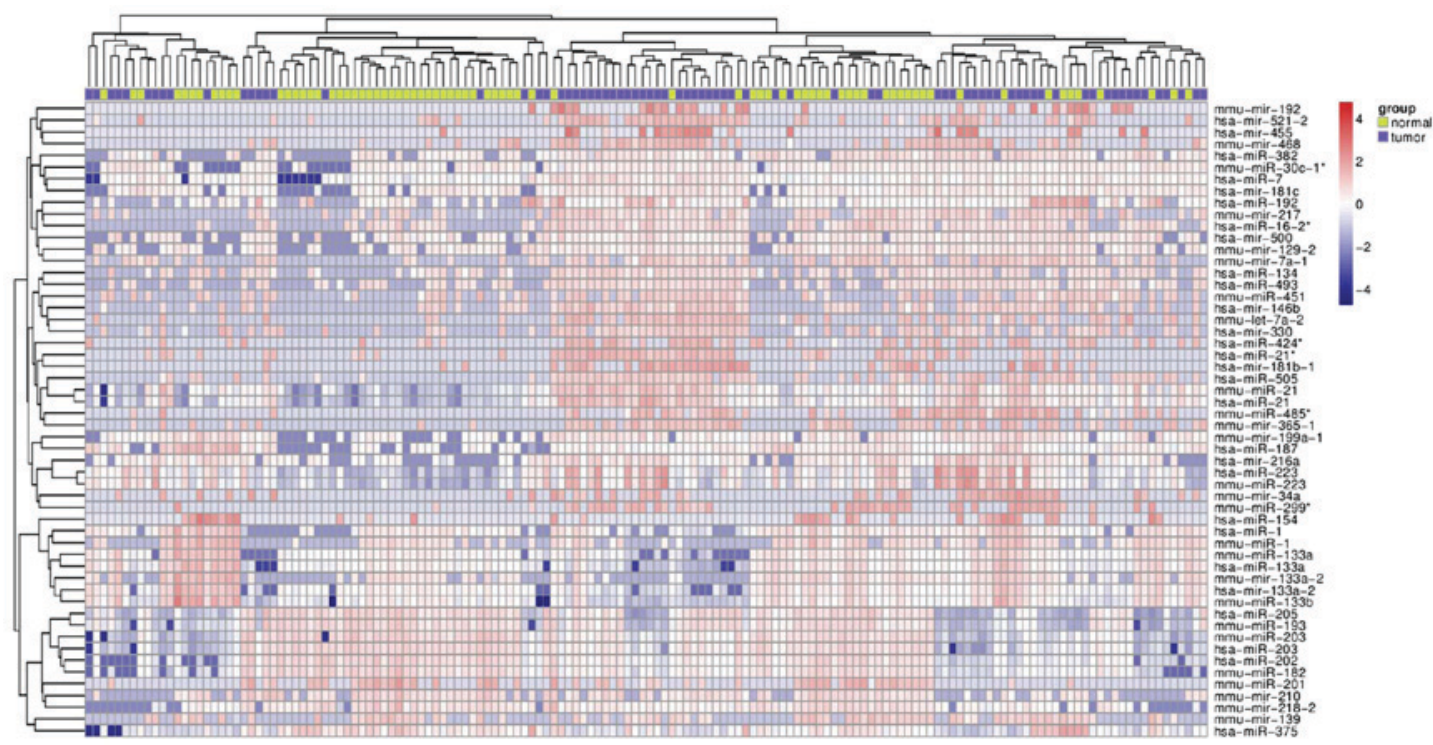

Figure 2. Heat map of the differentially expressed genes in carcinoma tissues and para-carcinoma tissues from 43 patients with esophageal squamous cell carcinoma and 32 patients with esophageal adenocarcinoma based on the GSE13937 microarray.

tissues. The top 10 upregulated and downregulated genes are presented in Table III.

Differentially expressed genes in common. In order to identify the differentially expressed genes in common, Venny was used. There were no upregulated genes in common among the three microarrays (Fig. 4A); however. there were two downregulated genes in common among the three microarrays, i.e., hsa-miR-1 and hsa-miR-203 (Fig. 4B). Subsequently, the expression of hsa-miR-1 and hsa-miR-203 in tumor tissue and normal tissue of patients was investigated, and it was identified that the expression of hsa-miR-1 and hsa-miR-203 in tumor tissue was decreased compared with that in normal tissue (Fig. 4C).
Target genes of hsa-miR-1 and hsa-miR-203. Following identification of the differentially expressed genes in common, the target genes of these differentially expressed genes was investigated. Venny was used to forecast the target genes of hsa-miR-1 and hsa-miR-203 based on the TargetScan, miRDB and PITA databases. As presented in Fig. 5A and B, respectively, 145 hsa-miR-1-targeted genes in common and 335 hsa-miR-203-targeted genes in common were identified. In order to narrow the number of these genes down further, protein-protein interaction networks were used to select the proteins located in the center of the networks. As presented in Fig. 6, proteins encoded by certain of the 145 genes exhibited numerous interactions and were located in the center of the 
Table II. Top 10 upregulated and downregulated genes in carcinoma cancer tissues and para-carcinoma tissues from 43 patients with esophageal squamous cell carcinoma and 32 patients with esophageal adenocarcinoma based on the GSE13937 microarray.

\section{A, Upregulation}

\begin{tabular}{lcl}
\hline Name & Log (fold-change) & P-value \\
\hline hsa-miR-192 & 1.981056158 & 0.003545 \\
mmu-miR-217 & 1.945886104 & 0.000282 \\
mmu-miR-199a-1 & 1.847687422 & 0.0004341 \\
hsa-miR-21* & 1.767488448 & 0.000499 \\
hsa-miR-505 & 1.746299252 & 0.0009254 \\
hsa-miR-146b & 1.745784019 & 0.0012439 \\
hsa-miR-134 & 1.681271144 & 0.0012697 \\
mmu-let-7a-2 & 1.681075105 & 0.0004522 \\
hsa-miR-330 & 1.674841191 & 0.0013867 \\
mmu-miR-485 & 1.63988565 & 0.0004462
\end{tabular}

B, Downregulation

\begin{tabular}{lll}
\hline Name & Log (fold-change) & P-value \\
\hline mmu-miR-133a & -2.353803547 & $4.89 \times 10^{-6}$ \\
mmu-miRr-210 & -1.974898362 & 0.000672 \\
hsa-miR-202 & -1.954744284 & 0.000222 \\
mmu-miR-203 & -1.913562145 & $3.34 \times 10^{-5}$ \\
hsa-miR-203 & -1.904073061 & 0.000361 \\
mmu-miRr-133a-2 & -1.81216527 & 0.003324 \\
mmu-miR-1 & -1.786346799 & 0.004887 \\
mmu-miR-201 & -1.786342985 & 0.000771 \\
hsa-miR-133a & -1.737590142 & $1.87 \times 10^{-5}$ \\
mmu-miR-182 & -1.724789689 & 0.00088
\end{tabular}

miR, microRNA; hsa, human; mmu, mouse.

protein-protein interaction networks, including RAB5A, RIT2, NRP1, EDN1, IGF1, KRAS, EIF4E, RPS6KA5, NOTCH2, NR3C1, PAX3, SFRP1, GJA1 and SNX2. Furthermore, as presented in Fig. 7, proteins encoded by certain of the 335 genes, exhibited numerous interactions and were located in the center of protein-protein interaction networks, including CPS1, MYO5, SRC, PRKCI, CLTC, DNM3, ADK, NEDD4L, PSMF1, GLI3, CNB4, PRKG1, MAD2L1, MYO6, CLASP2, GABBR2, PPP1R12A, SCN1A, PRKX, FMR1, CNOT6L, GABRB3, RERG, NEDD4L, GREM1, DCLK3, STYK1, SRC, GNA13, RERG, CLIC4, MEF2C and CORO1C. These genes are involved in certain pathways, including the cell adhesion pathway associated with tumor invasion, and were enriched in the interaction network of target genes of miR-1 and miR-203.

Further identification of the target genes of hsa-miR-1 and $h s a-m i R-203$. Following identification of the target genes of hsa-miR-1 and hsa-miR-203, the number of target genes was narrowed down further, by comparing the target genes with
Table III. Top 10 upregulated and downregulated genes in esophageal cancer tissue based on the GSE43732 microarray.

A, Upregulation

\begin{tabular}{lcc}
\hline Name & Log (fold-change) & P-value \\
\hline hsa-miR-99a-3p & 6.156811 & $2.03 \times 10^{-32}$ \\
hsa-miR-887 & 5.294203 & $9.04 \times 10^{-24}$ \\
hsa-miR-326 & 4.72286 & $2.52 \times 10^{-19}$ \\
hsa-miR-617 & 4.066399 & $1.46 \times 10^{-23}$ \\
hsa-miR-204-5p & 3.890677 & $7.13 \times 10^{-29}$ \\
hsa-miR-513b & 3.755658 & $2.41 \times 10^{-14}$ \\
hsa-miR-513c-5p & 3.733131 & $1.77 \times 10^{-16}$ \\
hsa-miR-378g & 3.538512 & $3.53 \times 10^{-19}$ \\
hsa-miR-4328 & 3.398278 & $1.32 \times 10^{-14}$ \\
hsa-miR-153 & 3.274099 & $2.16 \times 10^{-11}$ \\
\hline
\end{tabular}

B, Downregulation

\begin{tabular}{lcc}
\hline Name & Log (fold-change) & P-value \\
\hline hsa-miR-183-3p & -6.257640561 & $2.29 \times 10^{-50}$ \\
hsa-miR-503 & -6.212726325 & $1.57 \times 10^{-66}$ \\
hsa-miR-301b & -5.48315171 & $6.86 \times 10^{-41}$ \\
hsa-miR-542-5p & -5.077408003 & $3.18 \times 10^{-40}$ \\
hsa-miR-708-5p & -5.027636018 & $1.66 \times 10^{-28}$ \\
hsa-miR-106b-3p & -4.902767518 & $9.68 \times 10^{-28}$ \\
hsa-miR-421 & -4.447045181 & $4.75 \times 10^{-22}$ \\
hsa-miR-93-3p & -4.170004463 & $4.55 \times 10^{-18}$ \\
hsa-miR-542-3p & -4.164763727 & $1.94 \times 10^{-25}$ \\
hsa-miR-934 & -3.906983367 & $1.42 \times 10^{-19}$ \\
\hline
\end{tabular}

miR, microRNA; hsa, human.

GSE26886 microarray data. The GSE26886 microarray data displayed biomarkers to stratify patients with Barrett's esophagus in terms of risk of developing cancer. As presented in Fig. 8A and B, there were five upregulated genes of hsa-miR-1 in common with GSE26886, including MMD, BICD1, PTPRG, SDC2 and SEMA6D, and eight upregulated genes of hsa-miR-203 in common with GSE26886, including PXDN, NRCAM, FMNL2, EIF5A2, GLI3, FSL1, GREM1 and AHR (Table IV). Subsequently, the expression of these genes was investigated in tumor tissue and normal tissue, and it was identified that the expression of MMD, BICD1, PTPRG, SDC2, SEMA6D, PXDN, NRCAM, EIF5A2, GLI3, FSL1, GREM1 and AHR in tumor tissue was significantly increased compared with in normal tissue, and there was no statistical difference in the expression of FMNL2 between tumor tissue and normal tissue (Fig. 8C and D).

\section{Discussion}

Esophageal cancer is the eighth most frequently diagnosed type of cancer globally (1), and it is the sixth most 


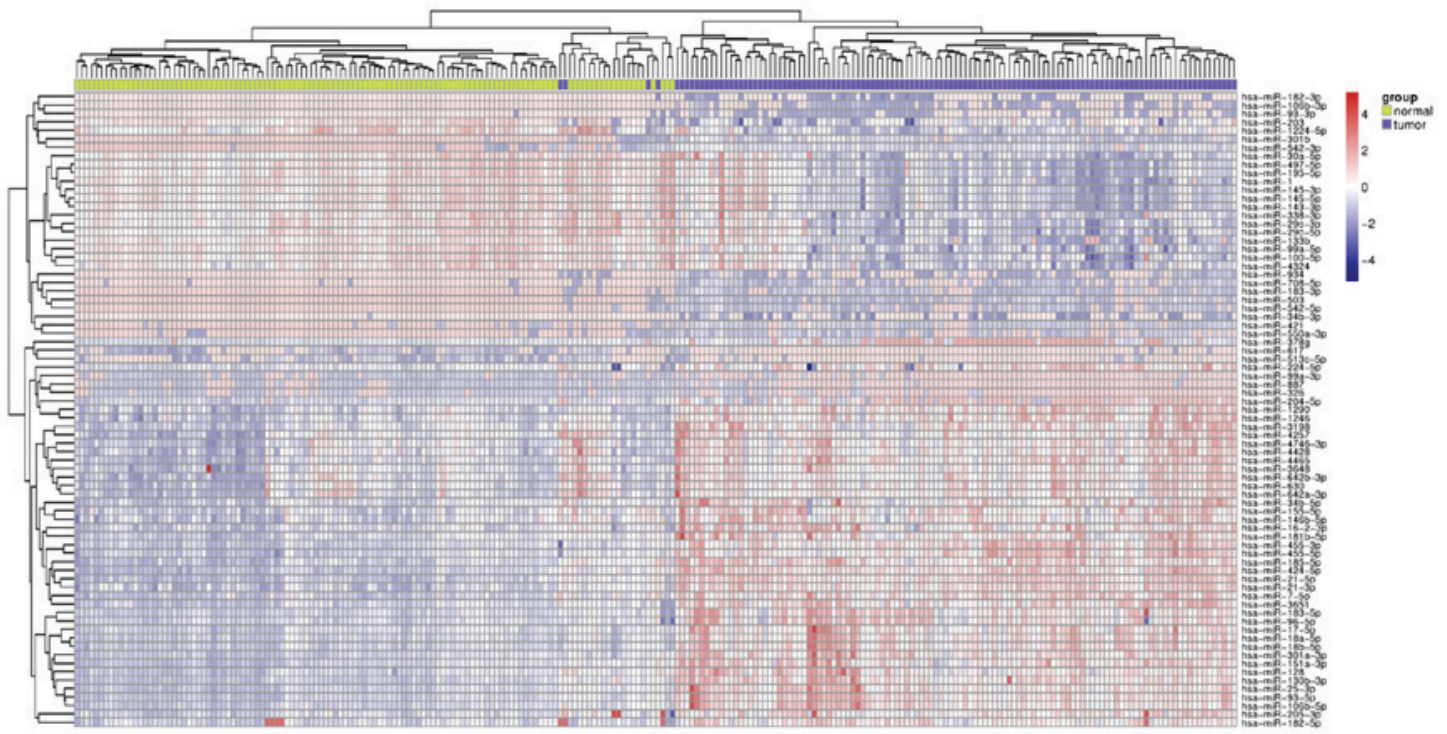

Figure 3. Heat map of the differentially expressed genes of esophageal cancer based on the GSE43732 microarray.

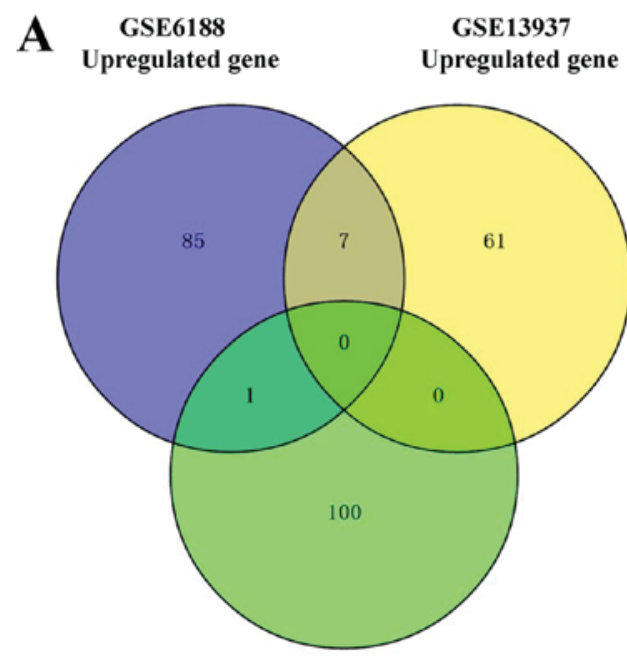

GSE43732

Upregulated gene

C

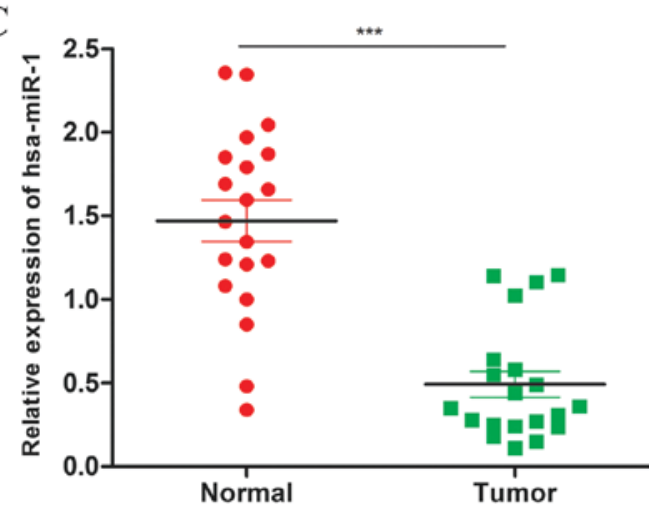

B $\begin{gathered}\text { GSE6188 } \\ \text { Downregulated gene }\end{gathered}$

GSE13937

Downregulated gene

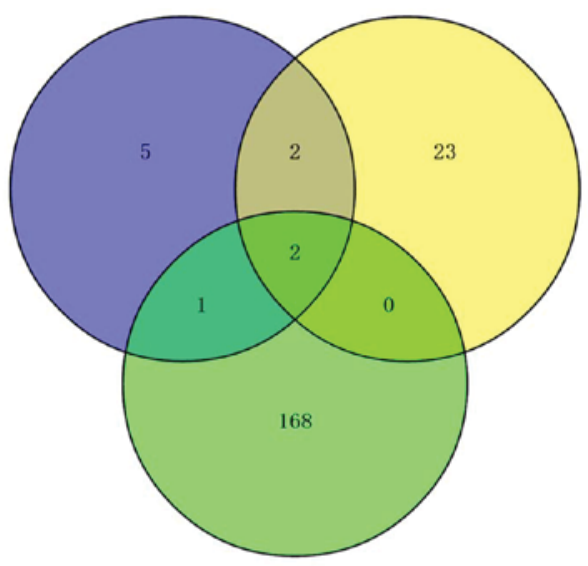

GSE43732

Downregulated gene

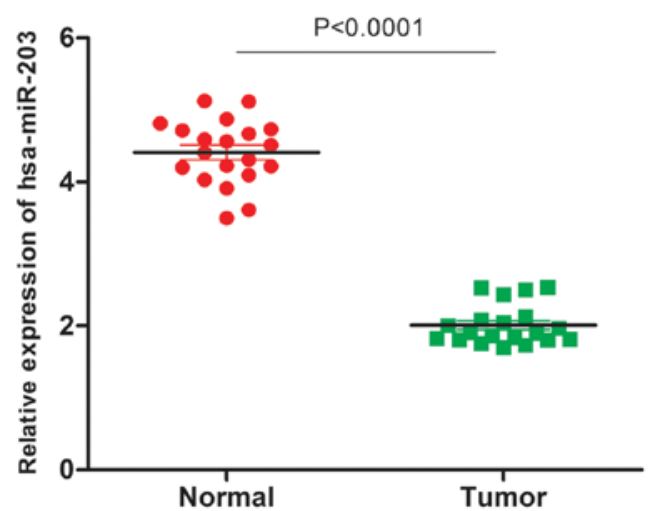

Figure 4. Upregulated and downregulated genes in common to the (Aand B) GSE6188, GSE13937 and (C) GSE43732 microarrays. hsa, human; miR, microRNA.

common cause of cancer-associated mortality due to its poor prognosis (17). It led to $\sim 400,000$ mortalities in 2012 , accounting for $\sim 5 \%$ of all mortality from cancer (1). In order to decrease the mortality rate, an improvement in diagnostic accuracy and more precise diagnostic markers are required.

miRNAs are able to decrease protein levels of their target genes with only a minor impact on the mRNA of the target 
A Tar-hsa-miR-1 miRDB-hsa-miR-1

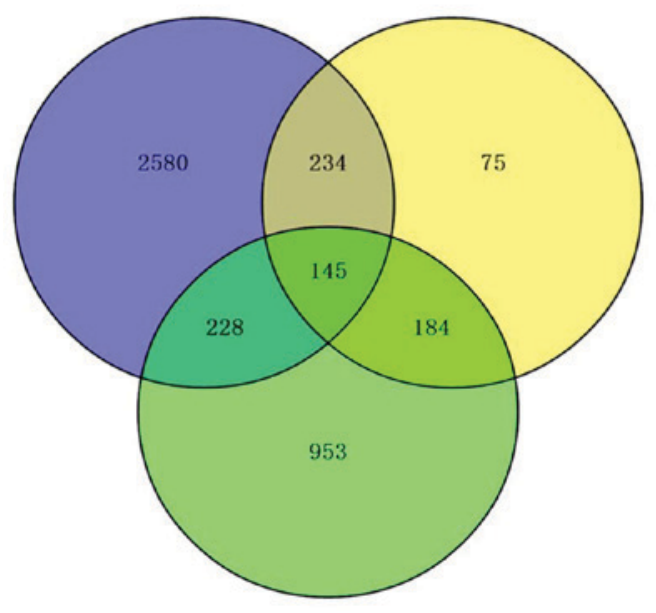

PITA-hsa-miR-1

hsa-miR-1 target genes
B Tar-hsa-miR-203 miRDB-hsa-miR-203

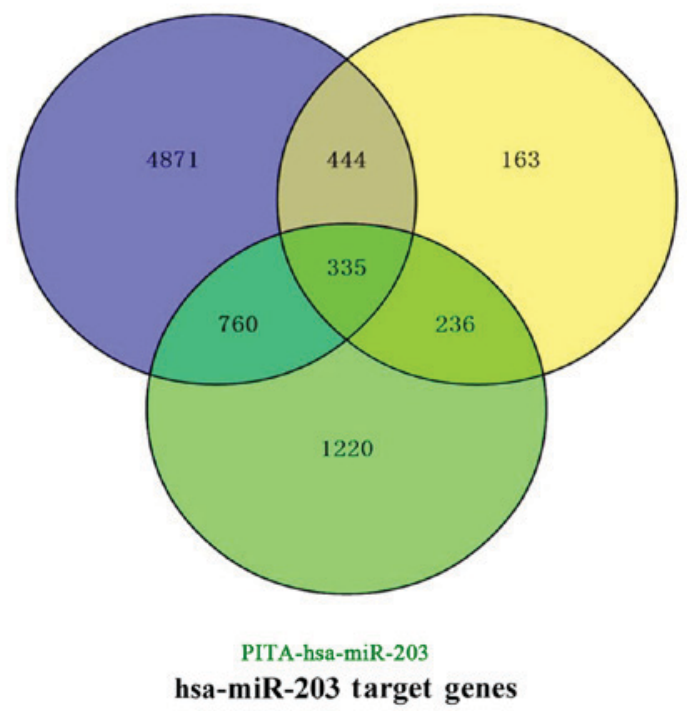

Figure 5. Target genes of (A) hsa-miR-1 and (B) hsa-miR-203 based on TargetScan, miRDB and PITA databases. hsa, human; miR, microRNA.

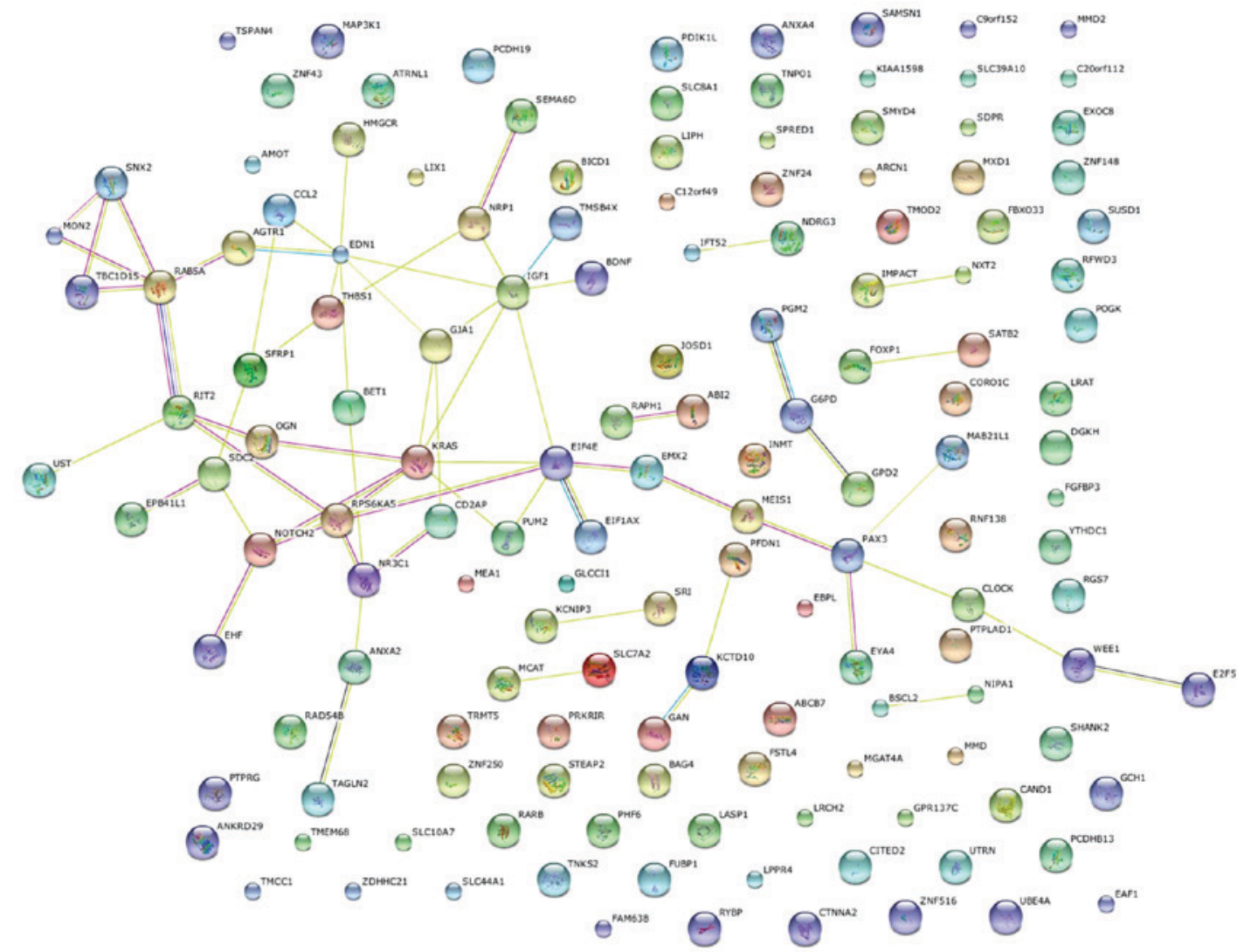

Figure 6. Protein-protein interaction network of predicted target genes of hsa-miR-1. The network indicates that the pathways with those genes, including the cell adhesion pathway associated with tumor invasion, were enriched in the interaction network of target genes of hsa-miR-1. hsa, human; miR, microRNA.

genes (18). miRNAs that are overexpressed in cancer may function as oncogenes, and miRNAs with tumor suppressor activity in normal tissue may be downregulated in cancer (19). Although major advances have been achieved in our understanding of cancer biology, as well as in the development of novel targeted therapies, progress in developing improved early diagnosis and screening tests has been inadequate.
There is therefore an intense research effort in seeking specific molecular changes that are able to identify patients with early cancer or precursor lesions. miRNA expression data in various types of cancer demonstrate that cancer cells have different miRNA profiles compared with normal cells; these unique properties of miRNAs make them useful potential agents for clinical diagnostics as well as in personalized 


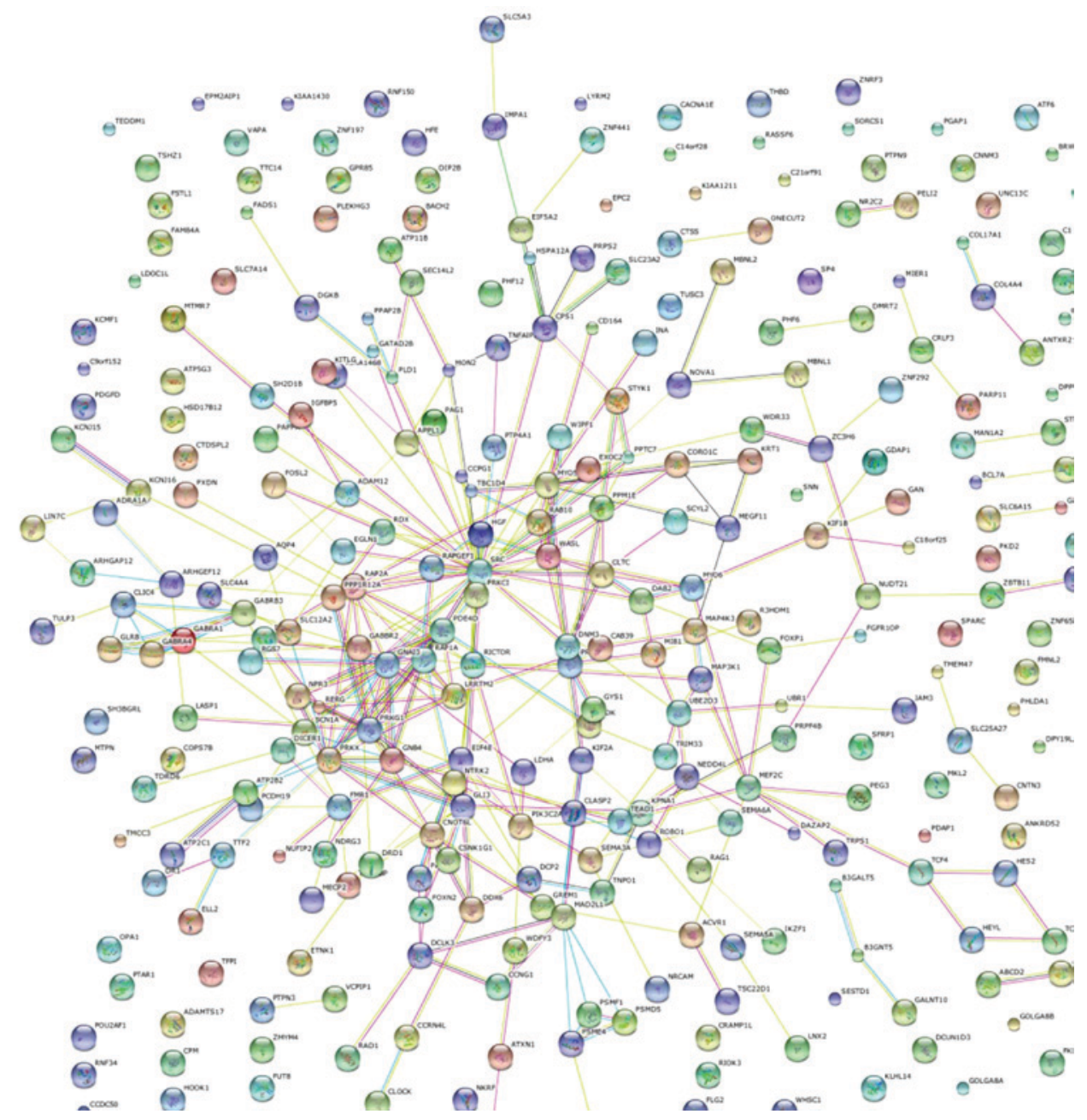

Figure 7. Protein-protein interaction network of predicted target genes of hsa-miR-203. The network indicates that the pathways with those genes, including the cell adhesion pathway associated with tumor invasion, were enriched in the interaction network of target genes of hsa-miR-203. hsa, human; miR, microRNA.
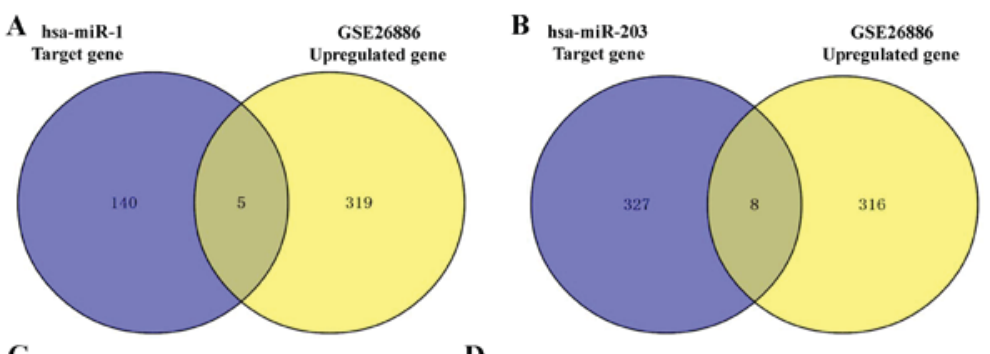

C

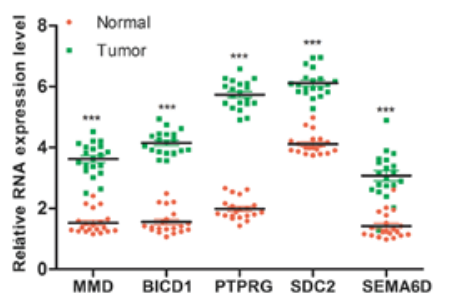

D

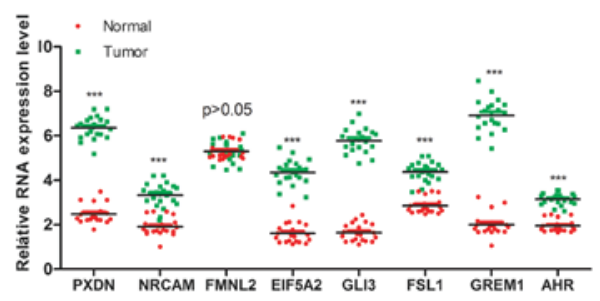

Figure 8. Upregulated genes in common between GSE26886 and hsa-miR-1, and between GSE26886 and hsa-miR-203. Upregulated genes in common between (A) GSE26886 and hsa-miR-1, and (B) GSE26886 and hsa-miR-203. Relative RNA expression levels of (C) the five genes in common between GSE26886 and hsa-miR-1, and (D) the eight genes in common between GSE26886 and hsa-miR-203.

care for individual patients in the future (20). In the present study, two important miRNAs (hsa-miR-1 and hsa-miR-203) were selected and their target genes which were differentially expressed in esophageal cancer were identified. The expression profiling may provide a useful lead for the diagnosis of esophageal cancer. 
Table IV. Upregulated genes in common between GSE26886 and hsa-miR-1 or hsa-miR-203 target genes.

A, hsa-miR-1

\begin{tabular}{lcc}
\hline Name & Log (fold-change) & P-value \\
\hline MMD & 2.146108 & $1.89 \times 10^{-9}$ \\
BICD1 & 2.080876 & $1.36 \times 10^{-9}$ \\
PTPRG & 2.196638 & $2.14 \times 10^{-8}$ \\
SDC2 & 2.185966 & 0.000237 \\
SEMA6D & 2.106877 & 0.00099 \\
\hline
\end{tabular}

B, hsa-miR-203

\begin{tabular}{lcc}
\hline Name & Log (fold-change) & P-value \\
\hline PXDN & 3.478027 & $2.21 \times 10^{-9}$ \\
NRCAM & 2.614851 & $5.24 \times 10^{-5}$ \\
FMNL2 & 3.281746 & $1.08 \times 10^{-12}$ \\
EIF5A2 & 2.688539 & $9.59 \times 10^{-9}$ \\
GLI3 & 2.026112 & $8.08 \times 10^{-9}$ \\
FSTL1 & 2.283896 & $4.40 \times 10^{-6}$ \\
GREM1 & 3.407262 & $5.67 \times 10^{-6}$ \\
AHR & 2.723882 & $4.68 \times 10^{-9}$ \\
\hline
\end{tabular}

miR, microRNA; hsa, human.

miRNAs are a class of regulatory RNAs that function primarily by targeting specific mRNAs for degradation or inhibition of translation and thus decrease the expression of the resulting protein, and their function in tumor development would presumably be through the regulation of their target protein genes $(21,22)$. In the present study, two downregulated miRNAs, hsa-miR-1 and hsa-miR-203, based on GSE6188, GSE13937 and GSE43732 microarrays were identified, whereas no upregulated miRNAs in common were identified. Previous studies reported that hsa-miR-1 and hsa-miR-203 were differentially expressed in cervical cancer (23), colorectal cancer (24), hepatocellular carcinoma (25) and lung cancer (26).

hsa-miR-1 is a muscle-enriched miRNA that inhibits the proliferation of progenitor cells and promotes myogenesis $(27,28)$. A number of key observations prompted the investigation of the antitumorigenic function of miR-1 in other tissues (29). For example, Nohata et al (30) revealed miR-1 to be a tumor suppressive miRNA targeting TAGLN2 in head and neck squamous cell carcinoma, and also demonstrated that the tumor suppressor miR-1 targets the oncogenic function of purine nucleoside phosphorylase in prostate cancer.

hsa-miR-203 has been identified as a skin-specific miRNA. It has also been identified to be differentially expressed in hepatocellular carcinoma (31) and leukemia (32). Furuta et al (31) proposed miR-203 as a tumor-suppressive miRNA in hepatocellular carcinoma (HCC) and hematopoietic malignancies. Bueno et al (32) also demonstrated silencing of miR-203 in certain types of leukemia. Supporting its function as a tumor suppressor, it has also been identified to be upregulated upon ultraviolet $\mathrm{C}$ irradiation in squamous cell carcinoma lines, suggesting an association between miR-203 and the activation of the apoptotic program (33).

The aforementioned studies demonstrated that hsa-miR-1 and hsa-miR-203 are able to function as tumor suppressive genes. Zang et al (34) demonstrated that hsa-miR-1 and hsa-miR-203 were downregulated in esophageal cancer, but the underlying molecular mechanism was not investigated. However, in the present study, it was demonstrated that hsa-miR-1 and hsa-miR-203 are downregulated in esophageal cancer, and the target genes of hsa-miR-1 and hsa-miR-203 were also identified. There were five upregulated genes of hsa-miR-1 in common with GSE26886, including MMD, BICD1, PTPRG, SDC2 and SEMA6D, and there were eight upregulated genes of hsa-miR-203 in common with GSE26886 including PXDN, NRCAM, FMNL2, EIF5A2, GLI3, FSL1, GREM1 and AHR.

The currently available methods for the diagnosis of cancer lack sufficient sensitivity and specificity to facilitate the detection of cancer in its early stages. In the case of esophageal cancer, the diagnostic tests available are invasive and uncomfortable for the patient, as well as expensive. The results of the present study demonstrated that a number of aspects of miRNAs including their intricate nature of interaction with multiple targets and multiple pathways make them useful potential agents for clinical diagnostics. However, there are limitations to the study. For instance, the databases used currently may not precisely forecast miRNAs, and meta-analysis with more datasets of gene expression analysis may be more reliable; however, meta-analysis requires a large number of clinical data, which are lacking. In future studies, meta-analysis should be used, if possible, to make an accurate prediction. Patient survival analysis is difficult to perform to validate the differentially expressed miRNAs and genes, as this requires monitoring patients with esophageal cancer following leaving the hospital.

\section{Acknowledgements}

Not applicable.

\section{Funding}

The present study was supported by the Shanghai Municipal Commission of Health and Family Planning (grant no. ZY-CCC-3-3045).

\section{Availability of data and materials}

The datasets generated and/or analyzed during the current study are available from the corresponding author on reasonable request.

\section{Authors' contributions}

$\mathrm{XC}$ and $\mathrm{XY}$ designed this study, MZ performed the bioinformatics analysis, CJ and LG perfomed the RT-PCR, LL, QC and XY made substantial contributions to acquisition of data, or analysis and interpretation of data, XC, YG and YD performed experiments and collected data. All authors have read and approved the manuscript. 


\section{Ethics approval and consent to participate}

Not applicable.

\section{Consent for publication}

Not applicable.

\section{Competing interests}

The authors declare that they have no competing interests.

\section{References}

1. International Agency for Research on Cancer (IARC): World Cancer Report 2014. Stewart BW and Wild CP (eds). IARC, Lyon, 2014. http://publications.iarc.fr/Non-Series-Publications/ World-Cancer-Reports/World-Cancer-Report-2014.

2. Ferri F: Ferri's Clinical Advisor 2014: 5 Books in 1. 1st edition. Mosby Elsevier, Philadelphia, PA, 2014.

3. Lozano R, Naghavi M, Foreman K, Lim S, Shibuya K, Aboyans V, Abraham J, Adair T, Aggarwal R, Ahn SY, et al: Global and regional mortality from 235 causes of death for 20 age groups in 1990 and 2010: A systematic analysis for the global burden of disease study 2010. Lancet 380: 2095-2128, 2012.

4. Enzinger PC and Mayer RJ: Esophageal cancer. N Engl J Med 349: 2241-2252, 2003.

5. Ambros V: The functions of animal microRNAs. Nature 431 350-355, 2004

6. Bartel DP. MicroRNAs: Genomics, biogenesis, mechanism, and function. Cell 116: 281-297, 2004.

7. Võsa U, Vooder T, Kolde R, Fischer K, Välk K, Tõnisson N, Roosipuu R, Vilo J, Metspalu A and Annilo T: Identification of miR-374a as a prognostic marker for survival in patients with early-stage nonsmall cell lung cancer. Genes Chromosomes Cancer 50: 812-822, 2011.

8. Akçakaya P, Ekelund S, Kolosenko I, Caramuta S, Ozata DM, Xie H, Lindforss U, Olivecrona $\mathrm{H}$ and Lui WO: miR-185 and miR-133b deregulation is associated with overall survival and metastasis in colorectal cancer. Int J Oncol 39: 311-318, 2011.

9. Jones K, Nourse JP, Keane C, Bhatnagar A and Gandhi MK: Plasma microrna are disease response biomarkers in classical hodgkin lymphoma. Clin Cancer Res 20: 253-264, 2014.

10. Wu $\mathrm{H}$ and Mo YY: Targeting miR-205 in breast cancer. Expert Opin Ther Targets 13: 1439-1448, 2009.

11. Gregory PA, Bert AG, Paterson EL, Barry SC, Tsykin A, Farshid G, Vadas MA, Khew-Goodall Y and Goodall GJ: The miR-200 family and miR-205 regulate epithelial to mesenchymal transition by targeting ZEB1 and SIP1. Nat Cell Biol 10: 593-601, 2008.

12. Hsu SD, Chu CH, Tsou AP, Chen SJ, Chen HC, Hsu PW Wong YH, Chen YH, Chen GH and Huang HD: miRNAMap 2.0: Genomic maps of microRNAs in metazoan genomes. Nucleic Acids Res 36: D165-D169, 2008.

13. Friedman RC, Farh KK, Burge CB and Bartel DP: Most mammalian mRNAs are conserved targets of microRNAs. Genome Res 19: 92-105, 2009.

14. Kozomara A and Griffiths-Jones S: miRBase: Integrating microRNA annotation and deep-sequencing data. Nucleic Acids Res 39: D152-D157, 2011.

15. Griffiths-Jones S, Saini HK, van Dongen S and Enright AJ: miRBase: Tools for microRNA genomics. Nucleic Acids Res 36: D154-D158, 2008.

16. Kertesz M, Iovino N, Unnerstall U, Gaul U and Segal E: The role of site accessibility in microRNA target recognition. Nat Genet 39: 1278-1284, 2007.

17. Zhang Y: Epidemiology of esophageal cancer. World J Gastroenterol 19: 5598-5606, 2013.
18. Guo H, Ingolia NT, Weissman JS and Bartel DP: Mammalian microRNAs predominantly act to decrease target mRNA levels. Nature 466: 835-840, 2010.

19. Paranjape T, Slack FJ and Weidhaas JB: MicroRNAs: Tools for cancer diagnostics. Gut 58: 1546-1554, 2009.

20. Gulyaeva LF and Kushlinskiy NE: Regulatory mechanisms of microRNA expression. J Transl Med 14: 143, 2016.

21. Bian Z, Li LM, Tang R, Hou DX, Chen X, Zhang CY and Zen K: Identification of mouse liver mitochondria-associated miRNAs and their potential biological functions. Cell Res 20: 1076-1078, 2010.

22. Li P, Jiao J, Gao G and Prabhakar BS: Control of mitochondrial activity by miRNAs. J Cell Biochem 113: 1104-1110, 2012.

23. Reshmi G and Pillai MR: Beyond HPV: Oncomirs as new players in cervical cancer. FEBS Lett 582: 4113-4116, 2008.

24. Corté H, Manceau G, Blons H and Laurent-Puig P: MicroRNA and colorectal cancer. Dig Liver Dis 44: 195-200, 2012.

25. Anwar SL, Albat C, Krech T, Hasemeier B, Schipper E, Schweitzer N, Vogel A, Kreipe H and Lehmann U: Concordant hypermethylation of intergenic microRNA genes in human hepatocellular carcinoma as new diagnostic and prognostic marker. Int J Cancer 133: 660-670, 2013.

26. Nasser MW, Datta J, Nuovo G, Kutay H, Motiwala T, Majumder S, Wang B, Suster S, Jacob ST and Ghoshal K: Down-regulation of micro-rna-1 (mir-1) in lung cancer: Suppression of tumorigenic property of lung cancer cells and their sensitization to doxorubicin-induced apoptosis by mir-1. J Biol Chem 283: 33394-33405, 2008.

27. Sokol NS and Ambros V: Mesodermally expressed Drosophila microRNA-1 is regulated by Twist and is required in muscles during larval growth. Genes Dev 19: 2343-2354, 2005.

28. Zhao Y, Samal E and Srivastava D: Serum response factor regulates a muscle-specific microRNA that targets hand2 during cardiogenesis. Nature 436: 214-220, 2005.

29. Mishima T, Mizuguchi Y, Kawahigashi Y, Takizawa T and Takizawa T: RT-PCR-based analysis of microRNA (miR-1 and -124) expression in mouse CNS. Brain Res 1131: 37-43, 2007.

30. Nohata N, Sone Y, Hanazawa T, Fuse M, Kikkawa N, Yoshino H, Chiyomaru T, Kawakami K, Enokida H, Nakagawa M, et al: miR-1 as a tumor suppressive microRNA targeting TAGLN2 in head and neck squamous cell carcinoma. Oncotarget 2: 29-42, 2011.

31. Furuta M, Kozaki KI, Tanaka S, Arii S, Imoto I and Inazawa J: miR-124 and miR-203 are epigenetically silenced tumor-suppressive microRNAs in hepatocellular carcinoma. Carcinogenesis 31: 766-776, 2010

32. Bueno MJ, Pérez de Castro I, Gómez de Cedrón M, Santos J, Calin GA, Cigudosa JC, Croce CM, Fernández-Piqueras J and Malumbres $\mathrm{M}$ : Genetic and epigenetic silencing of microRNA-203 enhances ABL1 and BCR-ABL1 oncogene expression. Cancer Cell 13: 496-506, 2008.

33. Lena AM, Shalom-Feuerstein R, Rivetti di Val Cervo P, Aberdam D, Knight RA, Melino G and Candi E: miR-203 represses' stemness' by repressing Np63. Cell death differ 15: $1187,2008$.

34. Zang W, Wang Y, Du Y, Xuan X, Wang T, Li M, Ma Y, Li P, Chen X, Dong Z and Zhao G: Differential expression profiling of microRNAs and their potential involvement in esophageal squamous cell carcinoma. Tumor Biol 35: 3295-32304, 2014.

This work is licensed under a Creative Commons Attribution-NonCommercial-NoDerivatives 4.0 International (CC BY-NC-ND 4.0) License. 